\title{
Reducing Measurement Error in Nutrition Assessment: Potential Research Implications for Iran
}

\author{
Yasmin Mossavar-Rahmani* \\ Department of Epidemiology \& Population Health, Albert Einstein College of Medicine, Bronx, New York, USA
}

\section{A B S T R A C T}

Self-reported measures of dietary intake are prone to measurement error that may obscure the relationship of diet and disease. This review addresses strategies to decrease errors during collection of dietary data and statistical approaches to deal with measurement issues once the data are collected. Examples from two US studies-- the Women's Health Initiative (WHI) Dietary Modification Trial and the Hispanic Community Health Study/Study of Latinos (HCHS/SOL) have been used.

Approaches to manage biases during the collection of data include home visits to assess actual portion sizes of foods consumed, early detection of random and systematic error, and technology-based tools to capture dietary intake. The discussion of the WHI and HCHS/SOL ancillary studies illustrates appropriate use of calibration and biomarkers. Biomarkers of interest include both recovery (doubly labeled water, urinary nitrogen, sodium, and potassium), and predictive biomarkers such as total sugars. Discussion of blood concentration biomarkers such as folate, tocopherols and carotenoids and novel biomarkers will supplement this review.

Once data are collected, biomarker calibration is an approach to deal with measurement error. Here the discussion of statistical methods includes the development of regression calibration equations that use both biomarkers and self-report measures along with pertinent participant characteristics; and hazard ratios (ratio of incidence rates) based on Cox regression for assessing diet-disease associations with biomarkers. This review concludes with approaches applicable to nutrition research in the context of Iran.

Keywords: Nutrition assessment, Measurement error, Biomarkers, $24 \mathrm{hr}$ dietary recall, Food frequency questionnaire, Diet records

\section{Significance and sources of biases in collection of dietary data}

Biases in dietary data collection can arise from several sources: diet assessment methodology, respondent's level of engagement with food preparation, interviewer's knowledge of local foods; and cultural norms regarding foods and health. Given the heterogeneity in cultural context, methodology and respondent's relationship with food, understandably there is a wide range of biases from one context to another (1). These biases distort the relationship between diet and disease (2-5). Measurement errors consist of random and systematic error. Random errors consist of those related to inattention such as failing to respond to random questions because of boredom or lack of interest (6), whereas systematic errors include those related to participant characteristics like body size or psychosocial characteristics (7). Attempts to reduce biases at the level of data collection have included training of diet interviewers to be non-judgmental, to be attuned to the respondents' emotional state, and to help the respondents focus during the data collection. For data collection via forms and questionnaires, colorful formatting and food images have been helpful (8).

Level of engagement with food: The respondents' and interviewers' lack of engagement with local foods and food preparation can impact bias; foods bought locally and prepared from scratch are less 
likely to be underreported. Interviewers, who are not familiar with local foods or how foods are named locally, may misinterpret the respondents' intake.

Cultural norms: Cultural norms can impact reporting of foods with socially desirable foods more likely to be reported. In a health conscious sample, foods perceived as unhealthy such as French fries and sweets could be underreported, and healthy foods such as fruits and vegetables could be overreported (9).

Memory and ability to describe foods: Memory and ability to describe foods on the part of the respondent affects accuracy of reporting. Even with use of food models and diagrams it may be a challenge for participants with low levels of numeracy and literacy to describe the spatial dimensions of foods. Strategies to overcome these challenges have included home visits where actual bowls and plates used can be measured; alternatively, participants have been asked to bring their own plates and bowls to the clinic interview (8).

Methodology: Methodology for dietary assessment such as type of administration (interviewer- or selfadministered) or format (food frequency or recalls) can impact bias. Participants with low levels of literacy may find it easier to speak to an interviewer, whereas literate participants may respond well to dietary intake administration via pencil and paper or web- or smart-phone-based modalities. The National Cancer Institute (NCI) in the US has created an automated self-administered web- and smartphonebased $24 \mathrm{hr}$ dietary recall (ASA-24) (10-11).

Twenty-four hour dietary recalls, food records and food frequency questionnaires (FFQ) can differ in level of completeness and hence bias. FFQs typically consist of a fixed list of foods designed to be representative of the foods consumed by population under study, and to assess diet over a length of time such as three months or a year. For populations with diverse culinary traditions, a multi-cultural FFQ might perform as opposed to a general FFQ designed for the mainstream population (12). The $24 \mathrm{hr}$ dietary recall can be self-administered or administered by an interviewer, and is used to assess dietary intake in many national surveys. When assessed during the week or week-end, it offers rich detail as to a respondent's usual intake with details such as timing of intake, type and amount of food, as well as source of foods consumed (13). For example, "Was the package of nuts purchased at the corner grocery store or from a vending machine?" With the addition of the food questionnaire (also known as food propensity questionnaire), information is added as to the frequency of consumption of episodic foods. This questionnaire assesses frequency (not amount) of episodically consumed foods that may not appear on the $24 \mathrm{hr}$ dietary recall. Two recent cycles of the National Health \& Nutrition Examination Survey (NHANES) in the US, a survey that assesses a nationally representative sample, have used the NCI method to estimate means and percentiles of the distribution of food intake. This method uses a $24 \mathrm{hr}$ dietary recall and a food questionnaire for episodically consumed foods to assess usual intake $(14,15)$. Lastly, food records (weighed or unweighed) provide detail usually over 3-7 days, and are completed by the participants; therefore, there is high participant burden associated with this method (16.) However, food records when conducted by respondents with a high level of interest in the task can result in records with a relatively high level of accuracy.

\section{So which is better: food record,}

\section{food frequency questionnaire or 24 hr diet recall?}

The answer depends on what is being assessed and for what purpose. Two US studies: the Women's Health Initiative (WHI) and the Hispanic Community Health Study/Study of Latinos (HCHS/SOL) offer examples. The landmark WHI study, a National Institutes of Health (NIH)-funded observational and clinical trial study of postmenopausal women in forty locations in the US, included biomarker studies to correct for error-prone self-report measures of diet $(2,3,17-20)$. The HCHS/SOL study, also an NIH funded study, is an epidemiological study of US Hispanics/Latinos in four communities in Bronx, Chicago, Miami, and San Diego. It also includes a biomarker study (5) to correct for self-report measures of diet (24 hr dietary recall and a food propensity questionnaire) (21-22). The respondents in both studies underreported energy intake by $25-32 \%$ when compared to the biomarker doubly labeled water; however, protein intake was underreported to a lesser extent (15-19\%) when compared to urinary nitrogen $(2,5)$. Underreporting was higher using the FFQ than $24 \mathrm{hr}$ 
dietary recall. On the other hand, potassium intake was less likely to be under-reported (23).

\section{Diet \& health outcomes-does}

\section{assessment modality bias the association?}

Assessment modality can impact ability to discern the association of diet with health outcomes. For example, the hazard ratio for breast cancer for each quintile increase of energy-adjusted fat was strongly associated with saturated fat intake measured using the food diary, but not the $F F Q$ in a prospective study relating diet to breast cancer risk in 13,070 women in the UK (24), indicating the advantage of using food records over FFQ in detecting an association with saturated fat intake and breast cancer. In the WHI biomarker studies, self-reported energy intake from four-day food records had a slightly higher correlation with the biomarker measure (doubly labeled water) than the $24 \mathrm{hr}$ dietary recall or the FFQ (see Figure 1) $(3,25)$. Nevertheless, all the three self-reported measures of intake seem to explain a relatively small amount of the biomarker variation for energy (2.8\% to $7.8 \%)$; as such, every study would benefit from the assessment of dietary intake both from biomarkers and self-report with respect to energy assessment. Understandably, this additional effort would add cost, so selection of the most practical modality would be important depending on the nutrient of interest. As an example, when assessing sodium intake, collection of spot or $24 \mathrm{hr}$ urine in a representative sub-sample could be used to develop calibration equations to correct error in the entire analytic sample (23).

\section{Established and new biomarkers}

Despite the attempts of the best interviewer, mismeasurement is inevitable; biomarkers can add important information in this regard (26). Table 1 shows the current state of diet-related biomarkers. These are markers in the blood, urine, breath, hair, nail or other biospecimens that can indicate an objective measure of the individual's level of nutriture that is closer to the "truth". Examples include the golden standard Doubly Labeled Water (DLW) to assess total energy expenditure and urinary nitrogen for protein (27). Other biomarkers are urinary sodium and potassium from $24 \mathrm{hr}$ urine samples. Sugar biomarkers such as fructose or sucrose are other biomarkers that can predict dietary intake (28-30). Sugar biomarkers typically require enzymatic assays or gas or liquid chromatography for measurement (31). Mass spectrometry is used for measurement of DLW, which is obtained from spot urine samples. Participants typically drink DLW based on their body weight; the amount of the biomarkers expended in urine from the DLW is then calculated using mass spectrometry to obtain an objective measure of total energy expenditure, which equals intake in weight stable individuals (32).

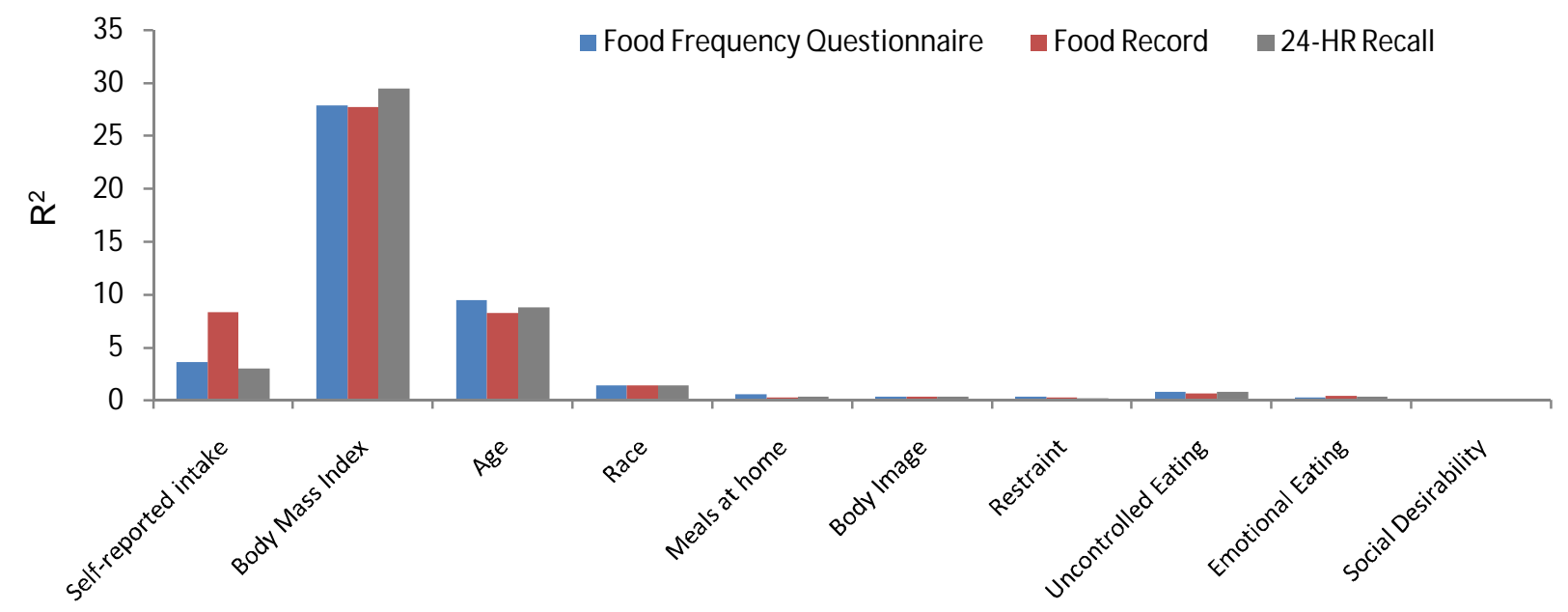

Figure 1. Fraction of variance in the biomarker (Doubly Labeled Water) explained by self-reported energy \& participants' characteristics.

Figure 1 adapted from: Mossavar-Rahmani Y, Tinker LF, Huang Y, Neuhouser ML, McCann SE, Seguin RA, Vitolins MZ, Curb JD, Prentice RL. Factors relating to eating style, social desirability, body image and eating meals at home increase the precision of calibration equations correcting self-report measures of diet using recovery biomarkers: findings from the Women's Health Initiative. Nutrition Journal 2013;12(1):63 (16 May 2013). 
Table 1. Biomarkers for dietary assessment

\begin{tabular}{|c|c|c|}
\hline & Biomarker & Type \\
\hline Energy & Doubly labeled water & True gold standard: recovery biomarker \\
\hline Protein & Urinary nitrogen & Recovery \\
\hline $\begin{array}{l}\text { Carbohydrate: } \\
\text { fructose } \\
\text { sucrose }\end{array}$ & $\begin{array}{l}\text { Total sugars: } \\
\text { Urinary fructose, sucrose from } 24 \mathrm{hr} \\
\text { urine }\end{array}$ & Predictive \\
\hline Fat & Biomarkers needed & \\
\hline Sodium & Urinary sodium from $24 \mathrm{hr}$ urine & $\begin{array}{l}\text { Replacement/data bases not available or incomplete; salt at } \\
\text { table difficult to quantify (other examples: phytoestrogens) }\end{array}$ \\
\hline Potassium & Urinary potassium from $24 \mathrm{hr}$ urine & Recovery \\
\hline Folate, carotenoids, tocopherols & Serum or levels in red blood cells & Blood concentration \\
\hline Added sugars & Carbon isotope ratios from fingerstick & Exploratory \\
\hline Intake of vegetable vs. animal protein & $\begin{array}{l}\text { Carbon, nitrogen isotope ratios from } \\
\text { hair and nails }\end{array}$ & Hair/nails \\
\hline Herbs and spices & Biomarkers needed & \\
\hline Polyphenols & Urine & Exploratory \\
\hline
\end{tabular}

Blood concentration biomarkers such as folate, carotenoids, tocopherols present challenges when correlating with intake because, unlike recovery biomarkers such as DLW, the amount ingested is typically not fully recovered in blood, and genetics or health conditions can all affect the blood levels of these nutrients. Feeding studies can provide insights into the relationship of these nutrients and their serum or plasma levels. Carbon and nitrogen isotope ratios from hair and nails are novel methods to assess vegetable versus animal sources of protein (33). Carbon isotope ratios from a fingerstick can also be used as a biomarker for added sugars' intake (34). Polyphenols are anti-oxidant rich plant based metabolites found in fruits, vegetables, legumes, and coffee/tea that have been associated with reduced risk of cardiovascular diseases (CVDs) (35). Finally, while biomarkers exist for energy, protein and other nutrients discussed in this review, novel and noninvasive biomarkers, especially for fat intake, are needed to better understand the relationship of diet to disease.

\section{Two applications of biomarker- calibrated energy intake}

a. Measurement properties of diet assessment tools and energy assessment: In the WHI study discussed earlier, recovery biomarkers were used to assess differences in the measurement properties of different diet assessment tools (3). Eating out frequently, being Black or Hispanic/Latino, and scoring high on the social desirability scale were significant predictors for underestimation of energy intake using the FFQ, but not for using the four-day food record nor the $24 \mathrm{hr}$ recall when assessed against the DLW biomarker (25). This finding reflects cognitive difficulties and biases inherent in the FFQ, a tool that uses a fixed list of foods; whereas a $24 \mathrm{hr}$ dietary recall or food record is cognitively easier for the respondent because, in contrast to a fixed list of foods typical of an FFQ, no assumptions are made as to what foods are eaten $(8,16)$. On the other hand, BMI was a significant predictor for underestimating energy from the $24 \mathrm{hr}$ recall and four-day food record, but not the FFQ. This finding brings up an interesting point that correlates related to misreporting may not necessarily be the same among the diet assessment tools, especially if one tool in particular is less sensitive to the respondents' food universe as compared to another assessment modality.

The $24 \mathrm{hr}$ dietary recall is used in the HCHS/SOL study. It is interviewer-administered, and is thought to have lower participant burden in a population for 
whom English is not the primary language (22). Figure 1 portrays the fraction of variance in the biomarker DLW explained by self-reported energy intake and participant characteristics from the WHI FFQ.

\section{b. Biomarker-calibrated energy and association} with health outcomes: An important application of biomarker-calibrated intake is its correlation with outcomes such as disease or functional status. Using Cox regression, calibrated energy consumption was found to be positively related to the risks of various CVDs (e.g. coronary artery bypass graft, coronary heart disease, coronary death, heart failure, myocardial infarction, percutaneous coronary intervention, stroke (hemorrhagic, ischemic)), invasive cancers (bladder, breast, colon, endometrial, kidney, leukemia, lung, lymphoma, ovarian, pancreatic, rectal) and diabetes in the WHI. Estimated hazard ratios for $20 \%$ increase in total energy consumption were: 1.49 (95\% CI: 1.18, 1.88) for total cardiovascular disease, 1.43 (95\% CI: 1.17, 1.73) for total invasive cancers; and 4.17 (95\% CI: $2.68,6.49)$ for diabetes (36). These associations were not evident in most corresponding analyses that had not been corrected for measurement error (1). Other studies have investigated the association of calibrated protein with physical function in the same cohort of post-menopausal women (37).

\section{Potential research implications for Iran}

As evidenced in this review, careful dietary data collection using home visits and understanding of respondents' food universe and attention to random and systematic error can potentially mitigate errors that could arise with less careful assessment. Table 2 shows steps for managing bias in data collection and analysis. In the context of Iran, women tend to be the primary individuals purchasing and preparing food and have intimate knowledge of the details of food preparation with recipes handed down mostly orally from one generation to the next. However, as more women work and eat outside the home, there is increased potential for misreporting and measurement error. Iranian men, on the other hand, may have more difficulty reporting the ingredients in foods eaten as they may be less familiar with food preparation. Assistance from the family member who prepares food and, where possible, direct observation of eating episodes in the preliminary stages of the assessment would be helpful in obtaining a better measure of the food universe. In addition, mixed dishes (stews or "khoreshts") with intricate combination of ingredients consisting of herbs or fruits and vegetables with considerable regional differences may be difficult to describe.

Children and adolescents may also have different reporting patterns with children needing help from parents to report intake and adolescents having eating patterns that are less structured. Adolescents may be more receptive to diet assessment using technology-based features such as smartphones. Language use is also important. If the local dialect is not used or participants have difficulty with the primary language, there could be significant underreporting. Further research is needed to understand reporting bias in children and adolescents and whether these biases are universal.

Table 2. Steps for managing bias in diet assessment

1. What method is used: FFQ, $24 \mathrm{hr}$ recall, food records, and weighed records?

--Is the FFQ representative of foods consumed by the population under study?

2. If $24 \mathrm{hr}$ recall was interview-administered or web- or smartphone based, and what language was used?

3. How many measures of the diet assessments were taken? The more measurements, the closer the correlation with truth.

4. Does the self-report correlate with the biomarker ( 0.30 or higher $)$ ?

5. Does the nutrient correlate with energy? (Then energy adjustment is a potential solution to dealing with under- or overreporting). 
Given the high cost of biomarkers, a sub-study representative of the population where possible could provide critical information such as whether gender, BMI, or age or other participant characteristics impact misreporting and whether these factors need to be controlled when using self-report measures and relating to outcomes. Investigation of new biomarkers, especially with respect to fat intake and flavonoids such as anthocyanins (found in berries) (38) and the emerging field of metabolomics are new areas of research. With regard to discovery of novel biomarkers in the context of Iran, biomarkers relating to spices/herbs and variety of green herbs that are abundantly and uniquely used in dishes in Iran and relationship to health outcomes would be a significant contribution to this area of investigation.

In conclusion, dietary assessment can be considerably enhanced, and measurement errors can be reduced with careful understanding of the context during data collection and biomarker calibration during data analysis.

\section{Financial disclosure}

The author declared no conflict of interest.

\section{Funding/Support}

No funding is associated with writing this review.

\section{References}

1. Harrison GG, Galal OM, Ibrahim N, Khorshid A, Stormer A, Leslie J, Saleh NT. Underreporting of food intake by dietary recall is not universal: a comparison of data from Egyptian and American women. J Nutr 2000;130(8):2049-54

2. Neuhouser ML, Tinker L, Shaw PA, Schoeller D, Bingham SA, Horn LV, Beresford SA, Caan B, Thomson C, Satterfield S, et al. Use of recovery biomarkers to calibrate nutrient consumption self-reports in the Women's Health Initiative. Am J Epidemiol 2008;167(10):1247-59.

3. Prentice RL, Mossavar-Rahmani Y, Huang Y, Van Horn L, Beresford SA, Caan B, Tinker L, Schoeller D, Bingham S, Eaton CB, et al. Evaluation and comparison of food records, recalls, and frequencies for energy and protein assessment by using recovery biomarkers. Am J Epidemiol 2011;174(5):591-603.

4. Mossavar-Rahmani Y, Tinker LF, Neuhouser ML, Huang Y, Shaw PA, Beasley JM, Di C, Zheng C, Li W, Prentice RL. Women's Health Initiative Dietary Modification Trial: Update and Application of Biomarker Calibration to Self-Report Measures of Diet
\& Physical Activity. Edtion ed. In: Balakrishnan N, ed. Methods and Applications of Statistics in Clinical Trials. New Jersey: John Wiley \& Sons, 2014:931-44.

5. Mossavar-Rahmani Y, Shaw PA, Wong WW, SotresAlvarez D, Gellman MD, Van Horn L, Stoutenberg M, Daviglus ML, Wylie-Rosett J, Siega-Riz AM, et al. Applying Recovery Biomarkers to Calibrate Self-Report Measures of Energy and Protein in the Hispanic Community Health Study/Study of Latinos. Am J Epidemiol 2015;181(12):996-1007.

6. https://dietassessmentprimer.cancer.gov/glossary.html\#r andom_error. (Accessed 12/31/2016).

7. https://dietassessmentprimer.cancer.gov/glossary.html\#s ystematic_error. (Accessed 12/31/2016).

8. https://epi.grants.cancer.gov/diet/adi/thompson_subar_di etary_assessment_methodology.pdf. (Accessed 12/31/ 2016).

9. Pryer A, Vrijheid M, Nichols R, Kiggins M, Elliott P. Who are the "low energy reporters" in the dietary and nutritional survey of British adults? Int. J. Epidemiol. 26 (1997) 146-154.

10. Kirkpatrick SI, Subar AF, Douglass D, Zimmerman TP, Thompson FE, Kahle LL, George SM, Dodd KW, Potischman N. Performance of the Automated SelfAdministered 24-hour Recall relative to a measure of true intakes and to an interviewer-administered 24-h recall. Am J Clin Nutr 2014;100(1):233-40.

11. Internet: http://epi.grants.cancer.gov/asa24/ (accessed 7/20/2016).

12. Forsythe HE, Gage B. Use of a multicultural foodfrequency questionnaire with pregnant and lactating women. Am J Clin Nutr 1994;59(1 Suppl):203s-6s.

13. Subar AF, Freedman LS, Tooze JA, Kirkpatrick SI, Boushey C, Neuhouser ML, Thompson FE, Potischman N, Guenther PM, Tarasuk V, et al. Addressing Current Criticism Regarding the Value of Self-Report Dietary Data. J Nutr 2015.

14. Tooze JA, Midthune D, Dodd KW, Freedman LS, Krebs-Smith SM, Subar AF, Guenther PM, Carroll RJ, Kipnis V. A new statistical method for estimating the usual intake of episodically consumed foods with application to their distribution. J Am Diet Assoc 2006;106(10):1575-87.

15. Internet: http://epi.grants.cancer.gov/diet/usualintakes/ method.html (Accessed 12/31/2016).

16. Mann J, Bingham S. Dietary Assessment. Chapter 30. In: J. Mann \& S. Bingham eds. Essentials of Human Nutrition. Oxford University Press, 2012. p. 491-504. 
17. Howard BV, Van Horn L, Hsia J, Manson JE, Stefanick ML, Wassertheil-Smoller S, Kuller LH, LaCroix AZ, Langer RD, Lasser NL, et al. Low-fat dietary pattern and risk of cardiovascular disease: the Women's Health Initiative Randomized Controlled Dietary Modification Trial. JAMA 2006;295(6):655-66.

18. Beresford SA, Johnson KC, Ritenbaugh C, Lasser NL, Snetselaar LG, Black HR, Anderson GL, Assaf AR, Bassford T, Bowen D, et al. Low-fat dietary pattern and risk of colorectal cancer: the Women's Health Initiative Randomized Controlled Dietary Modification Trial. JAMA 2006;295(6):643-54.

19. Rossouw JE, Finnegan LP, Harlan WR, Pinn VW, Clifford C, McGowan JA. The evolution of the Women's Health Initiative: perspectives from the NIH. J Am Med Women's Assoc 1995;50(2):50-5.

20. Rossouw JE, Hurd S. The Women's Health Initiative: recruitment complete--looking back and looking forward. J Women's Health 1999;8(1):3-5.

21. Sorlie PD, Aviles-Santa LM, Wassertheil-Smoller S, Kaplan RC, Daviglus ML, Giachello AL, Schneiderman N, Raij L, Talavera G, Allison M, et al. Design and implementation of the Hispanic Community Health Study/Study of Latinos. Ann Epidemiol 2010;20(8):62941.

22. Siega-Riz AM, Sotres-Alvarez D, Ayala GX, Ginsberg M, Himes JH, Liu K, Loria CM, Mossavar-Rahmani Y, Rock CL, Rodriguez B, et al. Food-group and nutrientdensity intakes by Hispanic and Latino backgrounds in the Hispanic Community Health Study/Study of Latinos. Am J Clin Nutr 2014;99(6):1487-98.

23. Huang Y, Van Horn L, Tinker LF, Neuhouser ML, Carbone L, Mossavar-Rahmani Y, Thomas F, Prentice RL. Measurement error corrected sodium and potassium intake estimation using 24-hour urinary excretion. Hypertension 2014;63(2):238-44.

24. Bingham SA, Luben R, Welch A, Wareham N, Khaw KT, Day N. Are imprecise methods obscuring a relation between fat and breast cancer? Lancet 2003;362(9379):212-4.

25. Mossavar-Rahmani Y, Tinker LF, Huang Y, Neuhouser ML, McCann SE, Seguin RA, Vitolins MZ, Curb JD, Prentice RL. Factors relating to eating style, social desirability, body image and eating meals at home increase the precision of calibration equations correcting self-report measures of diet using recovery biomarkers: findings from the Women's Health Initiative. Nutr J 2013;12:63.
26. Hedrick VE, Dietrich AM, Estabrooks PA, Savla J, Serrano E, Davy BM. Dietary biomarkers: advances, limitations and future directions. Nutr J 2012;11:109.

27. Schoeller DA. Recent advances from application of doubly labeled water to measurement of human energy expenditure. J Nutr 1999;129(10):1765-8.

28. Tasevska N, Runswick SA, McTaggart A, Bingham SA. Urinary sucrose and fructose as biomarkers for sugar consumption. Cancer epidemiology, biomarkers \& prevention : a publication of the American Association for Cancer Research, cosponsored by the American Society of Preventive Oncology 2005;14(5):1287-94.

29. Tasevska N, Midthune D, Potischman N, Subar AF, Cross AJ, Bingham SA, Schatzkin A, Kipnis V. Use of the predictive sugars biomarker to evaluate self-reported total sugars intake in the Observing Protein and Energy Nutrition (OPEN) study. Cancer Epidemiol, Bio \& Prev 2011;20(3):490-500.

30. Beasley JM, Jung M, Tasevska N, Wong WW, SiegaRiz AM, Sotres-Alvarez D, Gellman MD, Kizer JR, Shaw PA, Stamler J, et al. Biomarker-predicted sugars intake compared with self-reported measures in US Hispanics/Latinos: results from the HCHS/SOL SOLNAS study. Public Health Nutr 2016:1-9.

31. Hedrick VE, Dietrich AM, Estabrooks PA, Savla J, Serrano E, Davy BM. Dietary biomarkers: advances, limitations and future directions. Nutr J. 2012 Dec 14;11:109. doi: 10.1186/1475-2891-11-109. Review.

32. Schoeller DA, Hnilicka JM. Reliability of the doubly labeled water method for the measurement of total daily energy expenditure in free-living subjects. The Journal of nutrition 1996;126(1):348S-54S.

33. Petzke KJ, Boeing H, Klaus S, Metges CC. Carbon and nitrogen stable isotopic composition of hair protein and amino acids can be used as biomarkers for animalderived dietary protein intake in humans. J Nutr 2005;135(6):1515-20.

34. Hedrick VE, Davy BM, Wilburn GA, Jahren AH, Zoellner JM. Evaluation of a novel biomarker of added sugar intake (delta 13C) compared with self-reported added sugar intake and the Healthy Eating Index-2010 in a community-based, rural U.S. sample. Public Health Nutr 2016;19(3):429-36.

35. Perez-Jimenez J, Hubert J, Hooper L, Cassidy A, Manach C, Williamson G, Scalbert A. Urinary metabolites as biomarkers of polyphenol intake in humans: a systematic review. Am J Clin Nutr 2010;92(4):801-9. 
36. Zheng C, Beresford SA, Van Horn L, Tinker LF, Thomson CA, Neuhouser ML, Di C, Manson JE, Mossavar-Rahmani Y, Seguin R, et al. Simultaneous Association of Total Energy Consumption and ActivityRelated Energy Expenditure With Risks of Cardiovascular Disease, Cancer, and Diabetes Among Postmenopausal Women. Am J Epidemiol 2014. Sep 1;180(5):526-35.
37. Beasley JM, Wertheim BC, LaCroix AZ, Prentice RL, Neuhouser ML, Tinker LF, Kritchevsky S, Shikany JM, Eaton C, Chen Z, et al. Biomarker-calibrated protein intake and physical function in the Women's Health Initiative. J Am Geriatr Soc 2013;61(11):1863-71.

38. Wallace TC, Giusti MM. Anthocyanins. Adv Nutr 2015;6(5):620-2. 